\title{
Fiber fluorescent spectroscopy
}

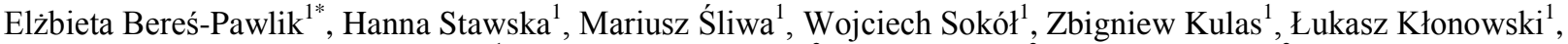 \\ Maciej Popenda ${ }^{1}$, Marcin Kochanowicz ${ }^{2}$, Jacek Żmojda ${ }^{2}$, Dominik Dorosz ${ }^{2}$ \\ ${ }^{I}$ Telecommunications and Teleinformatics Department, Wrockaw University of Technology, Wybrzeże \\ Wyspiańskiego 27, 50-370 Wrockaw, Poland, \\ ${ }^{2}$ Department of Power Engineering, Photonics and Lighting Technology, Faculty of Electrical Engineering, \\ Biatystok University of Technology, Wiejska 45D, 15-351 Bialystok, Poland
}

Received June 03, 2014; accepted June 28, 2014; published June 30, 2014

\begin{abstract}
In this paper we present the current history of fluorescent spectroscopy research conducted by our team. The results obtained by single-photon fluorescence spectroscopy are presented, together with a description of potential PCF fiber solutions for multi-photon type excitation. Single-photon research involved two methods: classica endoscope imaging and fluorescence lifetime analysis. The proposed PCF fibers are optimized for possible use as a fluorescent sensors, which will require the usage of femtosecond light pulses.
\end{abstract}

Fluorescent spectroscopy is known to be a very valuable tool when it comes to identifying elements, compounds etc. Its benefits are specificity for each of the analysed materials, simplicity of induction and relatively easy mechanism. Unsurprisingly, it is used so widely around the world. However, the introduction of other analytical and diagnostic methods, such as CT (computed tomography) or MRI (magnetic resonance imaging) puts it in a slight disadvantage. Nevertheless, it seems to be returning recently, especially in medical diagnostics.

Tissue fluorescence is used in medicine mainly for three purposes: the PDT (photodynamic therapy), PDD (photodynamic diagnosis) and autofluorescent diagnosis. The usual excitation wavelength is located in the blue-UV region ( $\lambda_{\text {ex }}$ ranges from $380 \mathrm{~nm}$ to $450 \mathrm{~nm}$ ), while the radiation response is from $\lambda_{\mathrm{r}}=350 \mathrm{~nm}$ to $\lambda_{\mathrm{r}}=635 \mathrm{~nm}$, with the maximum around 500nm. As already shown [1-3], green autofluorescence is weakened greatly in cancerous tissues. This fact was used during our investigation, as a numerical method of image analysis, optimized for the detection of 3 main colours - red, blue and green, was prepared (Fig. 1).

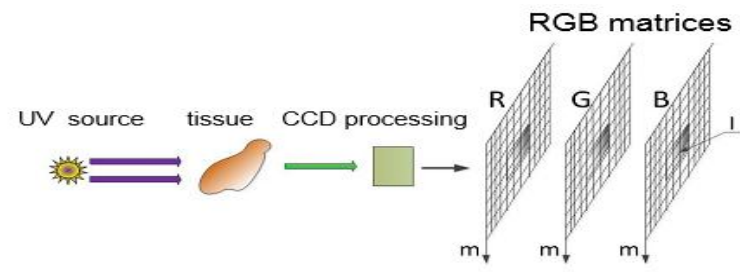

Fig. 1. The solution of CCD image analysis [4].
Tissues, observed and collected via CCD camera, were afterwards divided into pixels, for each of which we created a matrix of mentioned colours intensities, as it is presented in Fig. 2. The final results are in a form of endoscopic picture, imposed with a matrix of RGB colours intensities, which was a great help in the case of defining precisely the borders between a malignant and a normal tissue, all of which can be seen in [4].

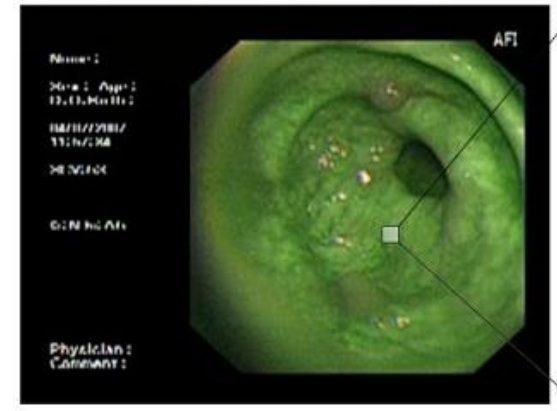

109105103

$R=109104104$

105100101

158154151

$\mathrm{G}=158152152$

154148149

$66 \quad 6565$

$B=67 \quad 66 \quad 66$

$65 \quad 62 \quad 63$

Fig. 2. Exemplary picture in the early stage of analysis. Three matrices $3 \times 3$ describe the $R, G, B$ colours intensities in the chosen pixel, spanning from 0 to 255 [4].

Another step of our investigation on the fluorescence of malignancies was the point fluorescence evaluation. Wide range excitation has its advantages, but is much more susceptible to events like quenching, concentration differences etc. Such a case requires small area excitation, which will result in a response signal of higher intensity. Obtaining this type of signal during medical procedures would be very difficult with the use of conventional optical devices, like collimators, lenses etc., because of their size and lack of mobility. Optical fibres provide the type of properties needed - high elasticity, combined with very good light propagation. Fibres are also thin enough to be introduced into the human body without any risk of harm. Together with small size optics, like GRIN lenses etc., they are a great tool for point - excitation of the tissue [5-7]. Their size also means that they are a great sensor material. In Fig. 3 an idea of such a sensor, designed for point excitation, is presented. The research over small area excitation of different types of tissue was 
later conducted with the use of a similar sensor; Fig. 4 presents the sensor measurement in a bronchoscope application setup. The light source was a laser diode, $\mathrm{CW}$ mode.

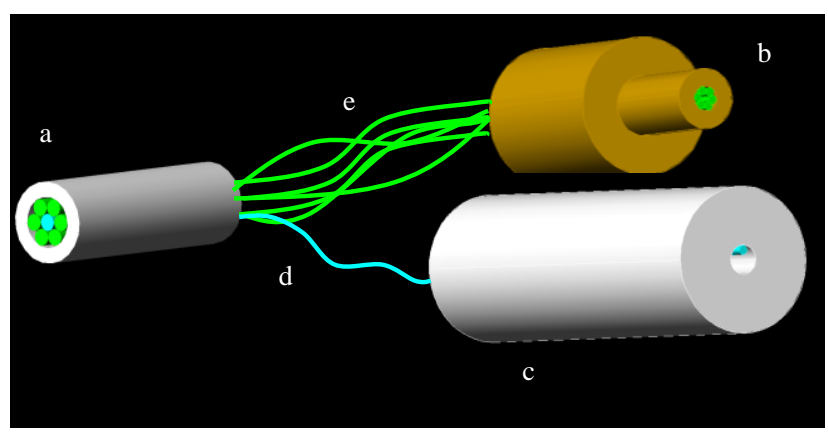

Fig. 3. Scheme of an optical fibre sensor. Efficiency is increased by the usage of few collecting fibres, while excitation is provided by the central one [8].

The average optical power of a laser beam was low enough not to damage the tissue, yet it allowed reliable excitation and provided interesting results. We were aware of the fact that fibers tend to fluoresce while being used for the propagation of blue and UV light. However, this phenomenon is significant mostly in the case of germanium fibres. In silica ones it is greatly reduced, and that is why we chose silica/silica fibres for this experiment. The use of a continuous wave laser allowed us to track the tissue response in real-time, which is an obvious advantage, especially as tissue samples have a short lifetime and change their response to excitation light over time. A more detailed description of the research can be seen elsewhere [6].

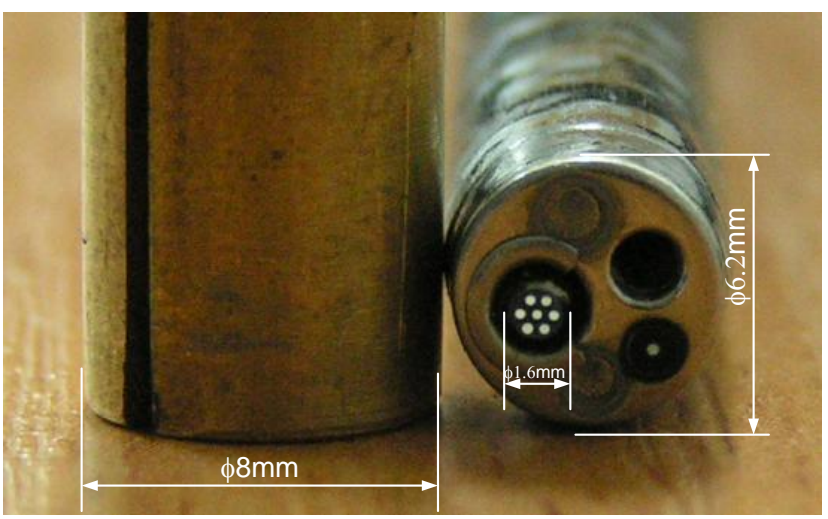

Fig. 4. Fibre sensor application [9].

Another step in improving the quality of point measurements was the introduction of fluorescence lifetime analysis. This method, due to its resistance to the aforementioned phenomena like quenching, compound concentration, etc., is the main element of time -resolved measurements as what we truly observed during the research was the time - intensity response of the fluorophore. The method itself is divided into two sub methods - the time domain and frequency domain measurements. The first one utilizes ultra - short laser pulses, resulting in pulsed fluorescence emission, which has the form of a decay curve. Data are then submitted for analysis which consists of proper fitting, allowing the direct determination of the fluorescence lifetime.
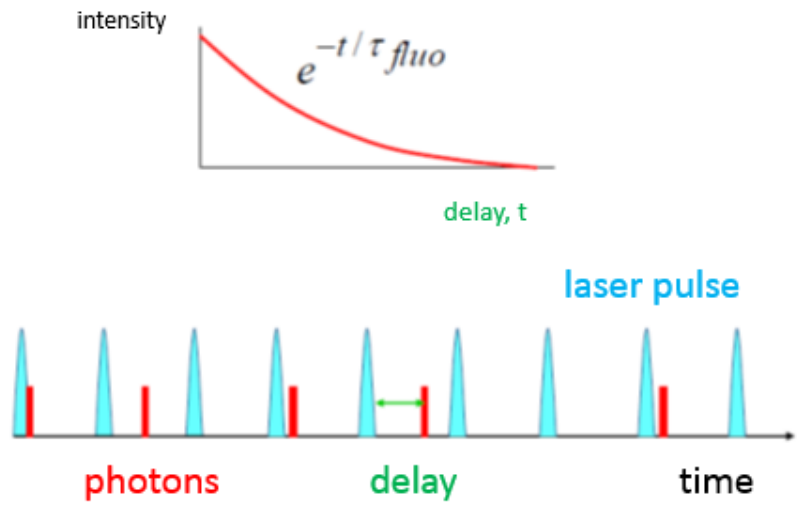

Fig. 5. Time domain fluorescence measurements principle. The method takes advantage of an exponential characteristics of the fluorescence decay [10].

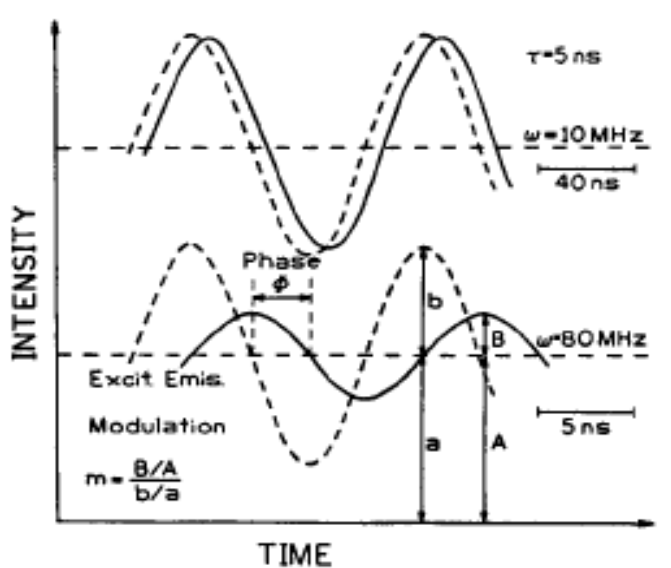

Fig. 6. Frequency domain measurements principle [11].

Frequency domain methods take advantage of intensity modulated lasers, and the fact that modulated excitation will result in demodulated emission. The demodulation character consists of the emission phase - shift and lower intensity when compared to the excitation light. Analysis of those factors allows later determination of the fluorescence lifetime. The principles of both methods are presented in Figs. 5-6.

The method of our choice was that of the time domain, as we were already in possession of a $407 \mathrm{~nm}$, pulsed laser source (TopGan). The pulse width (FWHM) was $\sim 50 \mathrm{~nm}$, repetition frequency $f_{\text {rep. }}=100 \mathrm{kHz}$ and power-per-pulse $\mathrm{P}_{\text {peak }}$ up to $1000 \mathrm{~mW}$ (depending on the repetition rate). 
Apart from the proper measurement setup, we have also prepared a fibre sensor, dedicated for point excitation measurements, which utilized the idea presented in Fig. 7. The idea of fluorescence lifetime measurements is an ongoing project in our team, already being investigated in terms of higher excitation pulse frequency and fibre optics usage.

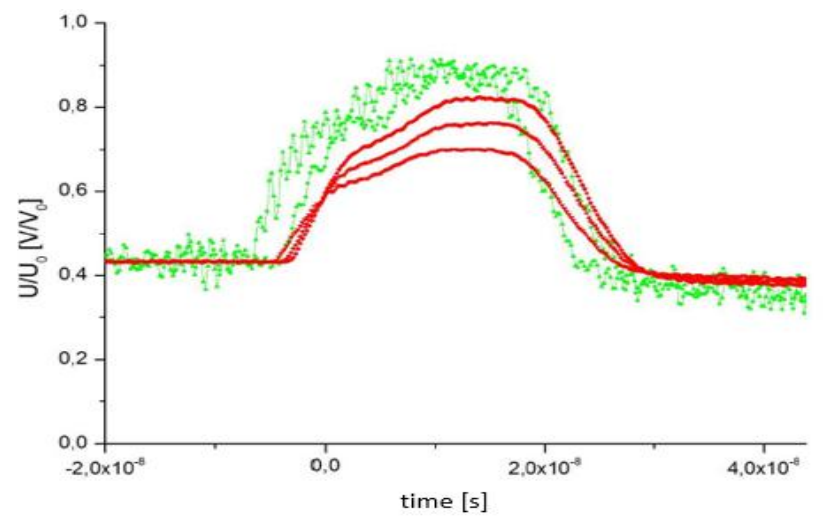

Fig. 7. Obtained results of time - to - intensity measurements of tissue fluorescence. The green and red curves represent a healthy and a malignant tissue, respectively. Signal intensity is defined as a ratio of sample signal voltage $(\mathrm{U})$ and detectors reference voltage $\left(\mathrm{U}_{0}\right)$ [10].

After some extensive work on single-photon excitation methods, we decided to take another step by introducing a little more up-to-date method, which is the multi - photon fluorescence. This field is relatively new, therefore it raises many possibilities of new discoveries, from signal introduction to the tissue, through its detection and further analysis. The principle of the method is similar to that of single photon fluorescence. However, the excitation process is a little different as there is more than one excitation signal required to rise the electron into a higher energy state. The difference is best seen in the fact that in single photon fluorescence, the exciting wavelength is shorter than the emission one, while in that of multi photon we have the opposite situation. Usually, both excitation photons have to be absorbed by the electron simultaneously for excitation to occur. This type of excitation, however, raises the problem of a reliable light source, for which the best would be a femtosecond pulsed laser at a wavelength of about $\lambda_{\mathrm{L}}=800 \mathrm{~nm}$. Wavelength elongation is allowed, as there will be two photons exciting the sample, which means their total energy will be equal to that of a $\sim 400 \mathrm{~nm}$ single photon. Femtosecond pulsed light laser sources, however, pose many problems when it comes to delivering such signals by the fibre. Classical double-clad fibres are characterized by high dispersion for signals of such energy; additionally, multiphoton methods use inconvenient bulk optics [12-13]. To avoid all those difficulties, our group proposed the project of a new PCF double - clad fibre, which can provide femtosecond signals with both low dispersion and attenuation. Eventually, we developed two different types of fibres - one with a hollow core, and one with a silica germanium - doped core. Both projects of such fibres, together with the results of simulated calculations of electric field distribution, losses and effective refractive index are presented in [14] and [15].

The fluorescent methods presented in this paper have a great potential in becoming a useful tool for oncologists and physicians, in general. Low cost, easy application, and high reliability in terms of measurement make them a very promising diagnostic tool. The increase of its potential was introduced with the use of optical fibres, which allowed easier in vivo imaging, because of their small size and ability to deliver a coherent light beam, as well as collect weak fluorescent light. Another described thing is the possibility to induce multi - photon excitation with the use of photonic crystal fibres. As our research shows, it is a viable solution for such a task, especially when considering wave beam requirements for such a method, and that is a femtosecond, pulsed light source. Great possibilities of PCF fibres, allowing us to collect more light, while being able to guide a pulse of a few hundred femtoseconds without any significant loss of its initial properties, make them a very important topic in our ongoing research.

\section{References}

[1] Z. Kulas, E. Bereś-Pawlik, T. Kręcicki, SPIE 59590 (2005).

[2] Z. Kulas, E. Bereś-Pawlik, T. Kręcicki, J. Non-Cryst Solids 32, 2468 (2006).

[3] E. Bereś-Pawlik, A. Grobelny, Z. Pałasz, T. Kręcicki, Polish Patent PL 207002 (2010).

[4] Z. Kulas, PhD Thesis, I28/2012/P-002, Wroclaw University of Technology (2012)

[5] U.S. Dinish, P. Gulati, V. M. Murukeshan, L.K. Seah, Opt. Comm. 271, 291 (2006).

[6] F.V. Bright, Ch.A. Munson, Analytica Chimica Acta 500, 71 (2003).

[7] G. Oh, E. Chung, S.H. Yun, Opt. Fiber Techn. 19, 760 (2013).

[8] E. Bereś-Pawlik, K. Gąsiorek, Z. Kulas, M. Rząca, Acta Phys. Polonica A 116, 254 (2009).

[9] K. Gąsiorek, M.S. Thesis, Wrocław University of Technology (2006)

[10] W. Sokół, M.S. Thesis, Wroclaw University of Technology (2013).

[11] B.B. Collier, M.J. McShane, J. Luminescence 144, 180 (2013).

[12] T. Dou et al., Opt. Expr. 18, 27900 (2010).

[13] S.F. Elahi, T.D. Wang, J. Biomed. Opt. 4, 471 (2011).

[14] H. Stawska, E. Bereś-Pawlik, Proc. 22nd International Conference on Optical Fiber Sensors, Beijing, China, OFS-22, 84217F-1 (2012).

[15] E. Bereś-Pawlik, H. Stawska, Ł. Kłonowski, Detecting cancerous tissues in human body by means of fiber fluorescent spectroscopy, ICTON (2013). 